\title{
Alzheimer's Disease and Musical Training: A Low-Cost Therapeutic?
}

\author{
Tiffany J. Ho \\ Cupertino High School, 10100 Finch Ave, Cupertino, CA, 95014, USA; tiffany.ho.cali@gmail.com
}

ABSTRACT: Alzheimer's disease (AD) affects over 50 million people worldwide and is the most prevalent neurodegenerative disease in the world. The CDC reported that in 2014, 5 million people were living with AD in the United States. This number has been projected to rise to 14 million by 2050 . AD is one of the top 10 leading causes of death in the United States; therefore, finding treatments and cures for these patients is crucial. Musical training has been observed to induce positive effects on brain development and plasticity, especially in regions of the medial temporal lobe. Providing a strong therapeutic approach in the improvement and restoration of neuroplasticity in the hippocampus, musical training holds the potential to alleviate neurological conditions in which the hippocampus is damaged, such as $\mathrm{AD}$. Here the potential of musical training as a low-cost medium with no known negative side effects by which patients may alleviate neurodegenerative symptoms is reviewed.

KEYWORDS: Behavioral and Social Sciences; Neuroscience; Alzheimer's Disease; Therapeutic; Musical Training.

\section{- Introduction}

Alzheimer's disease (AD), the most prevalent neurodegenerative disease and the most common form of dementia in the world, primarily affects adults over the age of 65. While the main risk factor of $\mathrm{AD}$ is age, other factors such as cardiovascular disease and genetics have been found to play significant roles in the pathogenesis of AD. Studies have also shown that $\mathrm{AD}$ is most notably associated with the formation of amyloid plaques, neurofibrillary tangles of tau protein, and hippocampal synapse loss. ${ }^{1}$ While different neurodegenerative diseases negatively impact different regions of the brain and, therefore, different cognitive functions, $\mathrm{AD}$ has shown the most prominent neuron loss in CA1 of the hippocampus. ${ }^{2}$ Other widely observed psychological symptoms of AD include depression, irritability, confusion, and paranoia. Nevertheless, the most common symptom of $\mathrm{AD}$ is memory loss. If damage to the hippocampus can be delayed or even restored, patients with AD may observe improved memory functions and reduced symptoms. Through neuronal networks connecting the auditory cortex and the hippocampus, musical training offers a potential medium by which hippocampal plasticity can be improved.

Through the stimulation of interconnected regions across the brain, music can alter brain function, generating a range of outcomes including lowered stress ${ }^{3}$ and feelings of excitement due to emotional arousal. ${ }^{4}$ Numerous networks work collaboratively to process and respond to musical inputs. These intricate connections allow for music's powerful effect on memory, learning, and emotion. ${ }^{5}$ Music first enters the brain through the ear in the form of sound waves. ${ }^{6}$ These sound waves hit the tympanic membrane, commonly known as the eardrum, causing a vibration that is transferred through the oval window and into the cochlea, a spiral-shaped cavity in the inner ear that resembles a snail shell. Higher frequencies with shorter wavelengths are responded to earlier compared to lower and longer wavelength frequencies, which are responded to farther along the cochlea. As vibrations pass through the cochlea, circular bundles of stereocilia-tiny hairs found on the inner surface of the cochlea-sway, allowing stimulatory neurotransmitters to flow from the Organ of Corti to the brainstem via the vestibulocochlear nerve. From the brainstem, signals are passed to the thalamus and on to the primary auditory cortex of the inner temporal lobe via neurons of the medial geniculate nucleus. Finally, at the auditory cortex, information is processed and sent to other brain regions for the formation of a response. The flow of this information to the hippocampus allows for the formation of auditory and musical memories.

The hippocampus is found in the medial temporal lobe of both hemispheres of the brain. Resembling the shape of a seahorse, the hippocampus consists of subregions including the cornu ammonis (CA), the dentate gyrus (DG), and the subiculum. The CA, shaped like rams' horns, can be further divided into four subregions called CA1, CA2, CA3, and CA4, each playing a distinct role in the brain's limbic system. Often recognized as a gateway and a relay station within the medial temporal lobe system, the entorhinal cortex (EC) has been found to convey highly processed sensory inputs from the neocortex into the hippocampus. ${ }^{7,8}$ It has also been shown that in the anterior temporal neocortex, a pathway involved in auditory object processing computes similarities and variations among the acoustic features of different instruments and objects. ${ }^{9}$ Through this pathway, auditory information is processed and sent to the hippocampus for long-term memory formation. Although the connectivity between the auditory cortex and the hippocampus is not yet fully understood, studies have suggested that the auditory cortex projects directly into the parahippocampal cortex, which is known to project into the posterior hippocampus. ${ }^{10}$ Neuronal connections between the auditory cortex and the hippocampus act as vehicles by which 
music and musical training can directly influence hippocampal function.

The hippocampus is involved in two main circuits: the trisynaptic circuit and the monosynaptic circuit. These circuits both allow information to flow from the EC to the hippocampus; however, the information that is passed differs. In the trisynaptic circuit, information is first passed from the $\mathrm{EC}$ to the DG via the perforant path. The s ignals $\mathrm{t}$ hen flow from the DG to CA3 via the mossy fiber pathway, and are finally transported from CA3 to CA1 along Schaffer collaterals. The monosynaptic circuit, on the other hand, transports information directly from the $\mathrm{EC}$ to $\mathrm{CA} 1$, providing a bypass circuit. In a 2015 study, Basu and Siegelbaum found that there is a 15-20 millisecond delay between the transmission of information through the trisynaptic circuit and the monosynaptic circuit. $^{11}$ Studies have also shown that CA1 integrates spatial and nonspatial sensory information in the trisynaptic circuit, suggesting that CA1 compares spatial sensory information and stored mnemonic information. This mechanical design allows for the integration of inputs that contribute to the encoding of episodic memory-the memory of experiences and past events.

Damage to or degeneration of the hippocampus can result in the inability to form new declarative memories. A widely known case of this defect was that of H.M., or Henry Molaison, who suffered epileptic seizures of the temporal lobe. An example of this is the case of the widely known patient H.M. or Henry Molaison, who suffered epileptic seizures of the temporal lobe. To treat his epilepsy and the induced seizures, Molaison had most of his hippocampus as well as surrounding parts of his temporal lobe removed in bilateral temporal lobe resection. As a result of this surgical procedure, Molaison lost the ability to form new episodic memories. Patients similar to Molaison were all severely impaired in the ability to form longterm memories, though most other cognitive functions, including short-term memory, seemed to be fully intact. ${ }^{12}$ This condition, known as anterograde amnesia, has been observed in $\mathrm{AD}$, traumatic brain injury, and several other neurological conditions. From these case studies, scientists have found that AD severely impacts both long-term and short-term memory through hippocampal deterioration.

Due to the intricate connections between the auditory cortex and the hippocampus, musical training has the capacity to influence the hippocampus. The hippocampus receives signals from the auditory cortex to form memories of timbres and pitch patterns, which allow most, regardless of musical experience, to recall songs and musical pieces they have heard before. When an auditory input is received and processed by the auditory cortex, its pitch, timbre, and duration are sent to the hippocampus where a memory of the sound is formed. Then, through the monosynaptic and trisynaptic circuits of the hippocampus, synaptic plasticity converts commonly heard sounds, such as voices and musical instruments, into long-term memory. ${ }^{2}$ The flow of information through these pathways allow for the activation of other structures in the brain such as the nucleus accumbens and amygdala, which are strongly associated with retrospection and emotional responses. ${ }^{13}$
Since AD most prominently affects CA1 of the hippocampus, ${ }^{14}$ musical training holds potential as a therapeutic by which hippocampal damage can be improved.

\section{- Discussion}

Hippocampal defects can severely affect one's cognitive abilities and quality of life. Due to hippocampal neuron loss in neurodegenerative diseases such as $\mathrm{AD}$, neurogenesis-the growth and development of new neurons in the brain-poses a potential solution. Neuropeptides including nerve growth factor (NGF) and brain-derived neurotrophic factor (BDNF) have been shown to facilitate neurogenesis, but this occurs at a rate much slower than that of hippocampal volume loss in moderate to severe AD. ${ }^{15}$ This poses a significant obstacle in the treatment of neurological conditions similar to $\mathrm{AD}$. If hippocampal plasticity can be improved, patients suffering hippocampal damage may lead improved lives. Therefore, further research on the mechanisms of hippocampal repair is vital to the development of more effective treatments for AD.

AD significantly affects memory, thinking, and other cognitive functions. Due to hippocampal atrophy, Alzheimer's patients often suffer memory loss and the inability to maintain new memories for long periods of time. In recent years, researchers have explored the effects of music on memory formation and retrieval, both of which are negatively impacted in AD. In 2010, Simmons-Stern and colleagues compared levels of memory encoding and recognition in Alzheimer's patients when presented with sung lyrics versus spoken lyrics. ${ }^{16}$ The research found that patients with $\mathrm{AD}$ observed a higher accuracy of lyric recognition when presented with sung lyrics compared to spoken lyrics. This supports that musical mnemonics-musical patterns used to aid in memorization-have the ability to help patients with AD learn new information. In addition, studies have investigated the connections between musical training and working memory (WM). Defined as short-term memory in which a limited amount of information is held in the brain for a short period of time, WM is crucial for the appreciation of musical experiences, as it allows for predictions of upcoming events. Differences between one's predictions and what is actually heard give music its ability to evoke memories and emotional responses. Scientists have discovered that musicians possess an enhanced ability to retrieve and process information using WM over non-musicians, ${ }^{17,18}$ suggesting that longterm exposure to musical training increases the plasticity of neuronal networks encoding auditory memory. From these studies, it is inferred that music offers a potential medium by which patients with $\mathrm{AD}$ may improve memory functions.

Diminished ability to form and retrieve memories in Alzheimer's patients is a direct result of grey matter volume loss in the hippocampus. In a 2014 study, musical training was found to increase the grey matter volumes of several brain areas in musicians. Groussard and colleagues observed that changes gradually appear in the hippocampus and several other regions of the temporal lobe and motor cortex during musical training. ${ }^{19}$ While neuroplasticity occurred in the left hippocampus and the right middle and superior frontal cortices shortly after engaging in musical training, 
modifications in the left posterior cingulate cortex, the superior temporal areas, the right supplementary motor area, and the insular cortex required several years of consistent training. Since all participants in the study were of the same age and education level, the observed results support that musical training induces structural changes in the brain, particularly the hippocampus. Further research is needed to establish how distinct brain regions and neural circuits are affected by musical training and whether Alzheimer'sinduced atrophy to the brain may be reduced or improved by musical training.

Potential of Musical Training as a Therapeutic for Alzheimer's Disease:

Due to the observed ability of musical training to enhance memory and improve plasticity in certain brain regions, researchers have begun exploring the effects of musical training on $\mathrm{AD}$. In a 2015 study, vocal training was evaluated for its effects on patients with mild to moderate AD. Patients in the experimental group attended 1-hour training sessions once a week for 6 months and were required to practice singing at least 3 times a week at home. From their research, Satoh and colleagues found that patients in the experimental group experienced improvements in psychomotor speed and decreases in symptoms of dementia after the 6-month vocal training program. ${ }^{20}$ In addition, a 2017 literature review supported that improved attention, memory, and executive functions were increasingly observed in $\mathrm{AD}$ patients after music therapy. ${ }^{21}$ Collectively, research suggests that musical training is associated with increased plasticity in motor and executive regions of the brain as well as decreased symptoms of dementia.

Previous studies concerning the effect of music on $\mathrm{AD}$ and related diseases have involved music listening and music therapy; however, the effects and efficacy of instrumental and vocal training have yet to be fully explored. With evidence that musical training improves hippocampal plasticity and other cognitive functions impaired in $\mathrm{AD}$, we propose to formally employ vocal and instrumental music training as a treatment option for improving Alzheimer's-induced hippocampal degeneration. Musical training using different instruments and training programs offers an unexplored research direction that may unlock new methods for alleviating symptoms of $\mathrm{AD}$ and other neurological disorders, producing a far-reaching impact on the millions of people living with AD.

\section{- Conclusions}

Studies show that musical training can improve hippocampal plasticity and increase grey matter volume in the hippocampus, presenting a potential treatment for Alzheimer's-induced hippocampal neuron loss. These improvements, resulting in refined WM, highlight the potential for therapeutic applications in neurological conditions that damage the hippocampus. Patients suffering from $\mathrm{AD}$ are a prime candidate for this therapeutic option, as they often observe impairments in both memory and hippocampal function. Studies have shown musical training to alleviate AD symptoms; however, the mechanisms underlying these benefits remain to be fully elucidated. Musical training sits poised as a potential therapeutic in the treatment of $\mathrm{AD}$ and associated neurodegenerative diseases.

\section{Acknowledgements}

I would like to thank Mr. Stephen Weber for his guidance and support in the preparation of this paper.

\section{References}

1. Scheff, S. W.; Price, D. A.; Schmitt, F. A.; Mufson, E. J. Hippocampal synaptic loss in early Alzheimer's disease and mild cognitive impairment. https://doi.org/10.1016/j. neurobiolaging.2005.09.012 (accessed Jul 1,2021).

2. Levitin, D. J. This is your brain on music; Plume Books: New York, New York, 2007.

3. Linnemann, A.; Ditzen, B.; Strahler, J.; Doerr, J. M.; Nater, U. M. Music listening as a means of stress reduction in daily life. https://doi.org/10.1016/j.psyneuen.2015.06.008 (accessed Jul 1, 2021).

4. Salimpoor, V. N.; Benovoy, M.; Longo, G.; Cooperstock, J. R.; Zatorre, R. J. The Rewarding Aspects of Music Listening Are Related to Degree of Emotional Arousal. https://doi. org/10.1371/journal.pone.0007487 (accessed Jul 1, 2021).

5. Lehmann, J. A. M.; Seufert, T. The Influence of Background Music on Learning in the Light of Different Theoretical Perspectives and the Role of Working Memory Capacity. https://doi. org/10.3389/fpsyg.2017.01902 (accessed Jul 1, 2021).

6. Peterson, D. C. Neuroanatomy, Auditory Pathway. https://www. ncbi.nlm.nih.gov/books/NBK532311/ (accessed Jul 1, 2021).

7. Canto, C. B.; Wouterlood, F. G.; Witter, M. P. What Does the Anatomical Organization of the Entorhinal Cortex Tell Us? https://doi.org/10.1155/2008/381243 (accessed Jul 1, 2021).

8. Schultz, H.; Sommer, T.; Peters, J. The Role of the Human Entorhinal Cortex in a Representational Account of Memory. https://doi.org/10.3389/fnhum.2015.00628 (accessed Jul 1, 2021).

9. Zatorre, R. J.; Bouffard, M.; Belin, P. Sensitivity to Auditory Object Features in Human Temporal Neocortex. https://doi. org/10.1523/JNEUROSCI.5458-03.2004 (accessed Jul 1, 2021).

10.Kumar, S.; Joseph, S.; Gander, P. E.; Barascud, N.; Halpern, A. R.; Griffiths, T. D. A Brain System for Auditory Working Memory. https://doi.org/10.1523/JNEUROSCI.4341-14.2016 (accessed Jul 1, 2021).

11.Basu, J.; Siegelbaum, S. A. The Corticohippocampal Circuit, Synaptic Plasticity, and Memory. https://doi.org/10.1101/ cshperspect.a021733 (accessed Jul 1, 2021).

12.Rubin, R. D.; Schwarb, H.; Lucas, H. D.; Dulas, M. R.; Cohen, N. J. Dynamic Hippocampal and Prefrontal Contributions to Memory Processes and Representations Blur the Boundaries of Traditional Cognitive Domains. https://doi.org/10.3390 brainsci7070082 (accessed Jul 1, 2021).

13.Cheung, V. K. M.; Harrison, P. M. C.; Meyer, L.; Pearce, M. T.; Haynes, J.-D.; Koelsch, S. Uncertainty and Surprise Jointly Predict Musical Pleasure and Amygdala, Hippocampus, and Auditory Cortex Activity. https://doi.org/10.1016/j.cub.2019.09.067 (accessed Jul 1, 2021).

14.Kril, J.J.; Hodges, J.; Halliday, G. Relationship between hippocampal volume and CA1 neuron loss in brains of humans with and without Alzheimer's disease. https://doi.org/10.1016/j. neulet.2004.02.001 (accessed Jul 1, 2021).

15.Peng, L.; Bonaguidi, M. A. Function and Dysfunction of Adult Hippocampal Neurogenesis in Regeneration and Disease. https:// doi.org/10.1016/j.ajpath.2017.09.004 (accessed Jul 1, 2021).

16.Simmons-Stern, N. R.; Budson, A. E.; Ally, B. A. Music as a memory enhancer in patients with Alzheimer's disease. https:// doi.org/10.1016/j.neuropsychologia.2010.04.033 (accessed Jul 1, 2021). 
17.Burunat, I.; Alluri, V.; Toiviainen, P.; Numminen, J.; Brattico, E. Dynamics of brain activity underlying working memory for music in a naturalistic condition. https://doi.org/10.1016/j. cortex.2014.04.012 (accessed Jul 1, 2021).

18.George, E. M.; Coch, D. Music training and working memory: An ERP study. https://doi.org/10.1016/j. neuropsychologia.2011.02.001 (accessed Jul 1, 2021).

19.Groussard, M.; Viader, F.; Landeau, B.; Desgranges, B.; Eustache, F.; Platel, H. The effects of musical practice on structural plasticity: The dynamics of grey matter changes. https://doi.org/10.1016/j. bandc.2014.06.013 (accessed Jul 1, 2021).

20.Satoh, M.; Yuba, T.; Tabei, K.-ichi; Okubo, Y.; Kida, H.; Sakuma, H.; Tomimoto, H. Music Therapy Using Singing Training Improves Psychomotor Speed in Patients with Alzheimer's Disease: A Neuropsychological and fMRI Study. https://doi. org/10.1159/000436960 (accessed Jul 1, 2021).

21.Fang, R.; Ye, S.; Huangfu, J.; Calimag, D.P. Music therapy is a potential intervention for cognition of Alzheimer's Disease: a minireview. https://doi.org/10.1186/s40035-017-0073-9 (accessed Jul 1, 2021).

\section{Author}

Tiffany Ho is a senior at Cupertino High School in Cupertino, California. She holds a deep passion for neuroscience and hopes to one day make a difference in the scientific community by conducting neurodegenerative disease research. She aims to pursue a career in clinical neuroscience. 